RAD Conference Proceedings, vol. 4, pp. 60-65, 2020

ISSN 2466-4626 (online) | DOI: 10.21175/RadProc.2020.12

www.rad-proceedings.org

\title{
RADIOSENSITIZING EFFECT OF BORON ENHANCES THE EFFECTIVENESS OF PROTON THERAPY IN VITRO
}

\author{
Dmitry Lebedevi,2, Luiza Garaeva1,2,3, Vladimir Burdakov',2, Andrey Volnitskiy 1,2, Natalya \\ Razgildina, ${ }^{1,2}$, Alina Garina1,3, Dmitry Amerkanov',2, Fedor Pack,1,2, Konstantin Shabalin',2, \\ Evgeniy Ivanov ${ }^{1,2}$, Victor Ezhov' ${ }^{1}$, Andrey Konevega ${ }^{1,2,3}$, Tatiana Shtam ${ }^{1,2 *}$
}

\author{
${ }^{1}$ Petersburg Nuclear Physics Institute named by B.P. Konstantinov of National Research Center "Kurchatov Institute”, \\ Gatchina, Russia \\ ${ }^{2}$ National Research Center "Kurchatov Institute", Moscow, Russia \\ 3Peter the Great St. Petersburg Polytechnic University, St. Petersburg, Russia
}

\begin{abstract}
Proton therapy is used today to treat many cancers and is particularly appropriate in situations where surgery options are limited, and conventional radiotherapy presents unacceptable risks to patients. A few years ago, it was suggested that an increase of up to a factor of two of the doses at the proton Bragg peak could be achieved if boron is accumulated in the tumor tissues. The mechanism responsible for a higher dose was suggested to be related to proton-boron fusion reactions, leading to the production of high Linear Energy Transfer (LET) $\alpha$-particles. Nowadays there are single works showing the effectiveness of proton beam irradiation boron-11-containing cancer cells. A limited number of the studies devoted to the application of $11 B(p, 3 a)$ nuclear reaction in proton therapy and lack of consistency in their results do not allow to judge about the prospects of the boron-containing drugs utilization in proton therapy to increase its antitumor efficacy. In this work, we experimentally test the possibility to enhance proton biological effectiveness in boron-11-containing cancer cells in vitro. Human glioblastoma cells were preincubated with boron compound ( $\mathrm{Na}_{2} \mathrm{~B}_{4} \mathrm{O}_{7}$, sodium tetraborate) and irradiated with increasing doses 2-8 Gy at the proton Bragg peak. To test whether the physical nuclear reaction $11 B(p, 3 a)$ results in an enhancement of the cancer cell death by high-energy proton beam irradiation, cell lines were also irradiated with graded doses 2-8 Gy using $\gamma$-ray source. The ability of boron compound to activate the cancer cell death with protons at the Bragg peak irradiation was shown in vitro. At the same time, weaker similar effect was determined for gamma-irradiation that may indicate not only the physical nature of influence boron at irradiated cancer cell viability but a specific biological effect. The data suggest that the combined effect of proton therapy with ${ }^{11} \mathrm{~B}$ on glioma cells increases their sensitivity to proton irradiation with low toxicity of the boron compound for cells of normal morphology.
\end{abstract}

Key words: Proton radiation therapy, Bragg peak, boron, radiosensitizer, proton boron capture therapy, glioma

\section{INTRODUCTION}

Proton therapy has a fundamental clinical advantage over conventional photon therapy that comes from the unique characteristics of the depth distribution of the absorbed dose of protons, which can be used both to achieve therapeutic dose values and to significantly reduce doses in normal tissues, proximal and distal to the target volume [1]. Protons quickly lose energy during the last few millimeters of tissue penetration, resulting in a sharply localized dose peak known as the Bragg peak. The Bragg peak can be precisely placed anywhere in the patient's body by modulating the proton energy, and several Bragg peaks can be displaced in depth to create a Spread-Out Bragg Peak (SOBP) that is used to irradiate the tumor.

Increasing the accuracy of proton targeting allows the implementation of technologies for increasing the radiation dose received by a tumor, increasing the biologically effective dose, reducing the physical dose of radiation to closely located critical organs of risk. The main difficulty of geometric targeting is the precise definition of the boundaries of the tumor and critical organs, which requires careful preliminary topometric preparation. Another limitation is that the specific features of the proton scattering characteristics do not allow achieving the required planning accuracy of the dose distribution. Changes in the patient's anatomy along the proton pathway can disrupt the conformity of dose distribution [2]. This is especially important for irradiating parts of the body where complex irregularities such as lung, head, neck are encountered, and where it is difficult to prevent large doses from being delivered to significant volumes of normal tissue distal to the target.

To radically increase the effectiveness of radiation therapy methods (photon and proton) there is a search for ways of modifying the radiosensitivity of normal and tumor tissues. Significant progress in this direction could be achieved as a result of the use of radiosensitizers [3,4]. Radiosensitizers are typically chemical or pharmacological agents that increase the probability of cell death from radiation. One of the options for radiosensitization is the introduction into the biological environment of elements with a significantly larger radiation absorption cross section than that of the biological tissue itself [5]. The resulting 
secondary short-range radiation localizes the absorption of energy near these elements and affects only the biological structures nearby, making it possible to locally increase the absorbed dose in the target. This approach defines the so-called "biochemical" targeting of radiation, which is a binary technology of radiation therapy, where targeting is performed to a greater extent by the increased concentration of the drug in the tumor, rather than geometric targeting of the radiation beam [6]. Binary technologies determined by the joint action of two components, i.e. radiation and drug are already used in clinical practice. These include, among others, boron neutron capture therapy (BNCT) [7] that uses drugs containing ${ }^{10} \mathrm{~B}$ isotope, and the additional absorbed dose is due to the products of nuclear reactions of thermal neutrons with this element.

Improving the therapeutic efficacy of proton therapy based on nuclear reactions, in an approach similar to that used in BNCT, is an attractive prospect. In 2014, the computational work was published [8], where the authors propose to use a unique exothermic nuclear reaction of protons on the boron-11 isotope to increase the absorbed dose in a tumor target with the formation of $\alpha$-particles: ${ }^{11} \mathrm{~B}+\mathrm{p} \rightarrow 3 \alpha$. The content of $11 \mathrm{~B}$ isotope in the natural isotopic mixture is approximately $80 \%$, which allows the use of biochemicals containing natural boron, thus avoiding expensive isotopic enrichment procedures. The cross section for proton capture reaction at proton energies common for Bragg peak is relatively large, reaching approximately 1 barn near $700 \mathrm{keV}$ (data of the TENDL-2017 library on the interaction of protons with various nuclides, https://tendl.web.psi.ch/tendl 2017 /tendl2017.html). This value, according to the authors [8], is an order of magnitude higher than the probability of other physical interactions of protons in a tissue-equivalent medium. A later computational work suggested that it is possible to ensure an almost two-fold increase in the absorbed dose at the Bragg peak at a boron concentration around $1000 \mathrm{ppm}$ and initial proton energy of $80 \mathrm{MeV}$ [9].

Recently, Cirrone and colleagues conducted in vitro study of the effect of sodium borocaptate (sodium mercapto-undecahydro-dodecaborate, $\mathrm{Na}_{2}\left[\mathrm{~B}_{12} \mathrm{H}_{11} \mathrm{SH}\right]$, $\mathrm{BSH}$ ) on the survival of DU145 prostate cancer cells, as well as on the frequency of chromosomal aberrations in MCF-10A breast cancer cells [10]. Irradiation of tumor cell cultures with protons was carried out at two concentrations of ${ }^{11} \mathrm{~B}$ in the nutrient medium, 80 and $40 \mathrm{ppm}$. It was shown that the presence of the $\mathrm{BSH}$ during proton irradiation can significantly reduce the survival of tumor cells and increase the number of chromosomal aberrations in comparison with control irradiation without the addition of BSH. However, several arguments cast doubt on the effectiveness of ${ }^{11} \mathrm{~B}$ in proton therapy. In the work by Mazzone A. et al. a more realistic assessment of a possible increase in the absorbed dose due to the proton capture reaction was carried out using the Geant4 program [11]. The calculated profiles of the absorbed dose along the proton track, taking into account all possible physical interactions of protons with the medium, including the interaction of protons with ${ }^{11} \mathrm{~B}$, show that the relative contribution from the reaction ${ }^{11} \mathrm{~B}+\mathrm{p} \rightarrow 3 \alpha$ at ${ }^{11} \mathrm{~B}$ concentration of $80 \mathrm{ppm}$ to the total absorbed dose is extremely small, approximately $10^{-7}$.

Taking into account the paucity of experimental studies in this area, the purpose of this work was to identify the sensitizing effects of boron compounds when exposed to protons in the Bragg peak on malignant cells cultivated in vitro.

\section{METHODS}

\subsection{Glioma cell lines and cultivation conditions}

The work was carried out using two human glioma cell lines: A172 cell line was obtained from the collection of the Institute of Cytology of the Russian Academy of Sciences (St. Petersburg, Russia) and glioma cell line (Gl-Tr) was generated in the Laboratory of Cell Biology (NRC «Kurchatov Institute»-PNPI, Gatchina, Russia) [12]. DF-2 fibroblasts of the skin of the eyelids of an adult donor were obtained from the collection of the Institute of Cytology of RAS were used as a control in cytotoxicity experiments. The cells were cultured in an atmosphere of $5 \% \mathrm{CO}_{2}$ at $37^{\circ} \mathrm{C}$ in DMEM/F12 (1:1) medium containing L-glutamine (BioloT, Russia), $0.1 \mathrm{mg} / \mathrm{ml}$ each of penicillin and streptomycin, and supplemented with $10 \%$ fetal bovine serum (BioloT, Russia).

\subsection{Analysis of cell survival and cell cycle parameters during incubation of glioma cells with sodium tetraborate}

To establish cytotoxicity of sodium tetraborate (Merck, USA), DF-2, A172, and Gl-Tr cells were plated at $5 \times 10^{3}$ cells/well in a 96-well tissue culture plate, incubated for 24 hours in complete medium, then the medium was replaced with a medium containing $\mathrm{Na}_{2} \mathrm{~B}_{4} \mathrm{O}_{7}$ in the concentration range $0-16 \mathrm{mM}$ (o $-640 \mathrm{ppm}$ ). After 18 hours of incubation, the medium was replaced with a complete one, and the cells were additionally incubated for 24 hours, after which the proliferative activity of the cells were tested

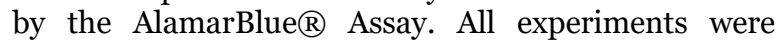
carried out in quintuplicate.

For the analysis of cell cycle, A172 and Gl-Tr cell lines were plated at $4 \times 10^{5}$ cells/well in a six well tissue culture plate, cultured for $24 \mathrm{~h}$, then the medium was replaced with a medium containing $2 \mathrm{mM}$ of $\mathrm{Na}_{2} \mathrm{~B}_{4} \mathrm{O}_{7}$ $\left(80 \mathrm{ppm}{ }^{11} \mathrm{~B}\right)$. After 18 hours of incubation in the presence of sodium tetraborate, the cells were trypsinized, washed with phosphate-buffered saline, and incubated for 10 minutes in a solution containing Triton X-100 (0.1\% solution v/v) and Hoechst 33342 $(10 \mu \mathrm{g} / \mathrm{ml})$. The fraction of cells in Go/G1, S and G2/M, based on Hoechst 33342 labelling of DNA content, was determined by flow cytometry (Beckman Coulter, USA). For each sample, $10^{4}$ events were collected. Experiments were performed in quintuplicate.

\subsection{Irradiation}

Two days before irradiation A172 and Gl-Tr cells

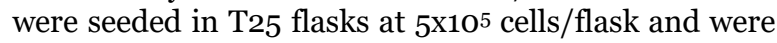
cultured for $24 \mathrm{~h}$. Then the cell growth medium was aspirated and replaced with $5 \mathrm{ml}$ of fresh medium 
(control) or with sodium tetraborate-containing medium. The working concentrations of $80 \mathrm{ppm}$ of ${ }^{11} \mathrm{~B}$ corresponded to $2 \mathrm{mM}$ of $\mathrm{Na}_{2} \mathrm{~B}_{4} \mathrm{O}_{7}$. After 18 hours of incubation in the presence of ${ }^{11} \mathrm{~B}$, the cells were trypsinized, washed with medium, resuspended in complete culture medium, and counted by LUNA-II ${ }^{\mathrm{TM}}$ Automated Cell Counter (Logos Biosystems, South Korea). Then each cell suspension was split into five portions (0.1 $\mathrm{ml}$ each), transferred into sterile Eppendorf tubes at $10^{5}$ cells/tube, and irradiated.

Proton irradiations were performed on $1 \mathrm{GeV}$ synchrocyclotron SC-1000 (NRC «Kurchatov Institute»-PNPI, Gatchina, Russia) using Roscosmos test facility (IS OP CDKT.412110.013) equipped with a variable length remote-controlled copper plate degrader and a bending magnet to reduce the energy spread [13]. The degrader length was set to $491 \mathrm{~mm}$ to generate a $200 \mathrm{MeV}$ proton beam with an energy spread of approximately $10 \mathrm{MeV}$. The beam had a round cross-section with diameter of $30 \mathrm{~mm}$ (intensity variations not exceeding 10\%). The beam was directed into a plexiglas tissue-equivalent phantom, resulting in a Spread-Out Bragg Peak (SOBP) located ca. $22 \mathrm{~cm}$ inside the phantom and spanning approximately 25 $\mathrm{mm}$ of the phantom depth. The phantom depth was varied by addition of the appropriate number of 2.8 $\mathrm{mm}$ plexiglass plates. The position of SOBP was determined by a series of dose rate measurements using Unidos Webline clinical dosimeter, which were also used for the dose calibration. The dose rate variations within SOBP did not exceed $10 \%$. The proton beam was calibrated before each experiment. Cell samples were positioned in the phantom near the middle of SOBP and irradiated with increasing doses of 2-8 Gy.

To test whether the physical nuclear reaction $11 \mathrm{~B}(\mathrm{p}, 3 \mathrm{a})$ results in an enhancement of the cancer cell death by proton beam irradiation, cell lines were also irradiated with graded doses 2-8 Gy using lift-up type ${ }^{60} \mathrm{Co} \gamma$-ray source "Issledovatel" (NRC «Kurchatov Institute»-PNPI, Gatchina, Russia).

\subsection{Cell viability and colony formation assays after irradiation}

For cell viability assay irradiated and control cells were seeded at $1 \times 10^{4}$ cells/well in a 24 well tissue culture plate and incubated at normal conditions. 48 hours after irradiation the cells were passaged at a concentration of $1 \times 10^{3}$ cells/well in 96 well tissue culture plate in five replicates of each experimental point. To determine the survival rate, the

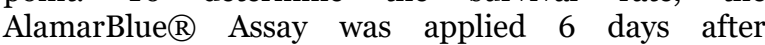
irradiation. AlamarBlue Cell Viability Reagent (Invitrogen) was added to the plate according to the manufacturer's protocol and incubated for two hours. The fluorescence was detected using EnSpire Multimode Plate Reader (PerkinElmer, USA).

Clonogenic assay is the method of choice to determine cell reproductive death after treatment with ionizing radiation. After irradiation, cells were seeded at $1 \times 10^{3}$ cells/well in a six well tissue culture plate, to form colonies in 1-2 weeks. Cells were incubated in standard conditions until cells in control dishes have formed sufficiently large clones. Then colonies were fixed with 96\% ethanol, stained with crystal violet $(0.5 \% \mathrm{w} / \mathrm{v})$, washed with water, dried in air, photographed and counted by ImageJ software.

\subsection{Statistical analysis}

Statistical analysis was performed using SPSS 17.0. To assess differences between groups, the Mann Whitney test was used, and the level of significance was set at $\mathrm{p}<0.05$. Experimental data are expressed as the mean \pm the standard deviation (SD).

\section{RESULtS}

The effect of boron on the viability of the cancer cells subjected to proton irradiation was assessed in vitro using two human malignant glioma cell lines, (A172 and Gl-Tr) and sodium tetraborate $\left(\mathrm{Na}_{2} \mathrm{~B}_{4} \mathrm{O}_{7}\right)$ as a boron containing substance at $2 \mathrm{mM}$ a concentration in the growth medium, that corresponds to $80 \mathrm{ppm}$ concentration of ${ }^{11} \mathrm{~B}$.
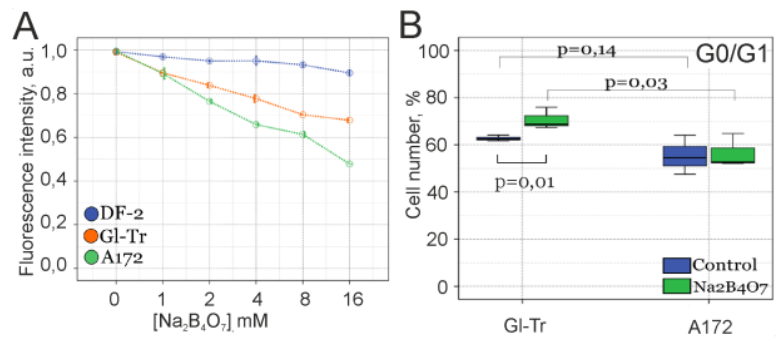

C

$$
\text { A172 }
$$

$\mathrm{Gl}-\mathrm{Tr}$
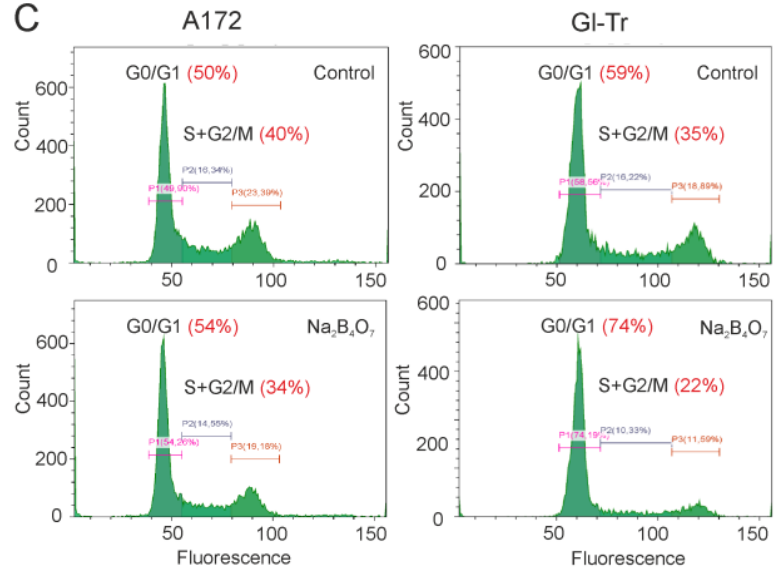

Figure 1. Effect of sodium tetraborate on proliferation and cell cycle distribution. (A) Viability of normal morphology DF-2 epithelial (blue line) and malignant A172 (green line) and Gl-Tr (red line) glioma cells incubated in media containing different concentration of sodium tetraborate. Data obtained by AlamarBlue Cell Viability assay. (B) The quantitative measurement of Go/G1 cell cycle phase of Gl-Tr and A172 glioma cells after incubation with $\mathrm{Na}_{2} \mathrm{~B}_{4} \mathrm{O}_{7}$. (C) A typical cell cycle analysis through Hoechst 33342 staining and following flow cytometry for A172 and Gl-Tr cells incubated in the presence of $\mathrm{Na}_{2} \mathrm{~B}_{4} \mathrm{O}_{7}$ (lower panels) or without it (control, upper panels).

Sodium tetraborate appears to be non-toxic for the normal morphology DF-2 epithelial cells. A noticeable cytostatic effect of sodium tetraborate was observed in both glioma cell lines. However, at the concentrations used in irradiation experiments it was relatively small: 
the decrease of the viable cells did not exceed $25 \%$ after 18 hours of incubation with $2 \mathrm{mM} \mathrm{Na} \mathrm{B}_{4} \mathrm{O}_{7}$ (Fig. 1 panel A).
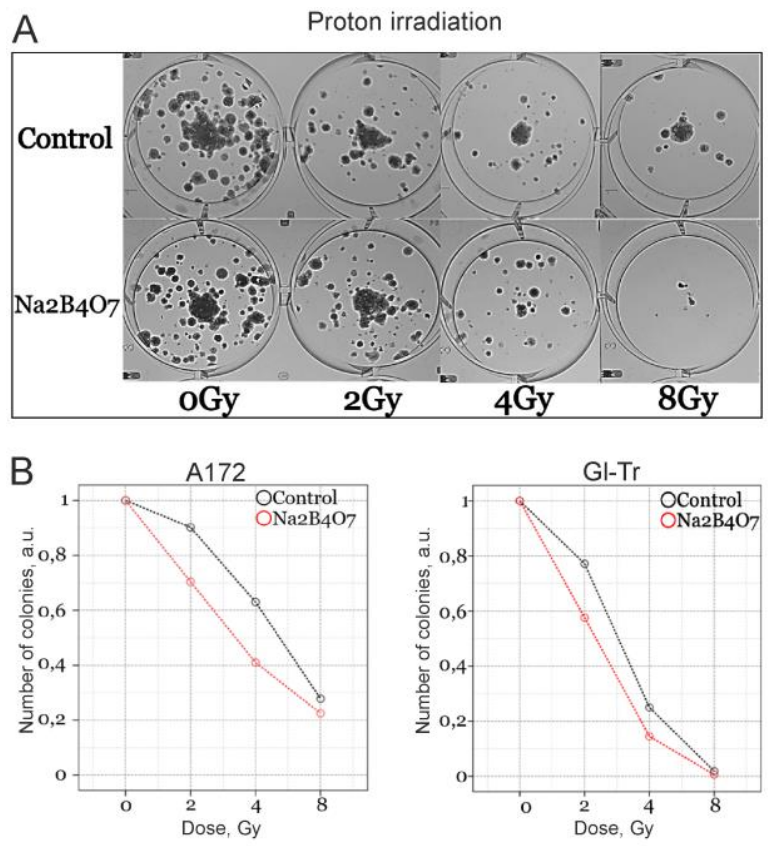

C
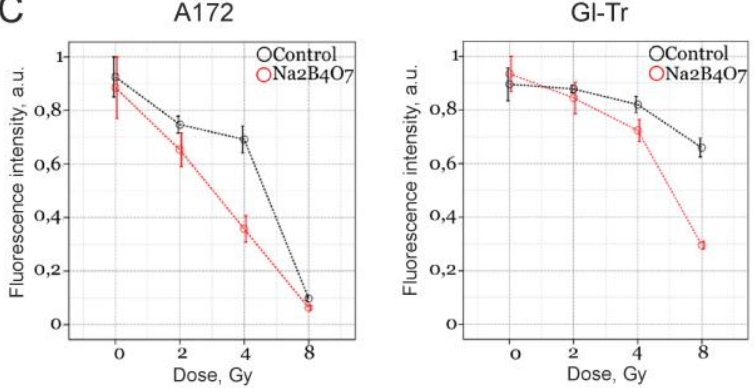

Figure 2. Effect of sodium tetraborate on cell survivability after exposure to Bragg peak protons at different absorbed dose. (A) A typical colony counting test pattern for A172 cell line. (B, C) Summary plots for clonogenic and AlamarBlue (analog of MTT-test) assays. Data were normalized to maximum values of the relevant control group.

The measurements of the distribution of the cultured cells through the cell cycle have shown a typical pattern with Go phase accounting for about two-thirds of the cells, while approximately $15 \%$ cells were in the $S$ phase, and the rest of the cells were in G2/mitotic phase. Notably, the Gl-Tr culture exhibited fewer G2/M and more Go cells than A172, particularly after incubation with $80 \mathrm{ppm} \mathrm{Na}_{2} \mathrm{~B}_{4} \mathrm{O}_{7}((71 \pm 3) \%$ vs.

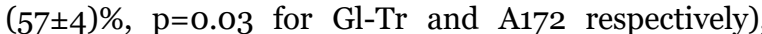
which may be important for its resistance to damage by ionizing radiation (Fig. 1 panels $\mathrm{B}$ and $\mathrm{C}$ ). No measurable effect of sodium tetraborate on cell cycle distribution was observed in A172 cells, while in Gl-Tr cells a significant increase (from $(63 \pm 1) \%$ to $(71 \pm 3) \%$, $\mathrm{p}=0.01)$ in the fraction of cells in Go phase was registered (Fig. 1 panels $\mathrm{B}$ and $\mathrm{C}$ ).

Cytotoxic effect of cell irradiation by Bragg peak protons on A172 cells was clearly seen by colony counting in the dose range 2 - $8 \mathrm{~Gy}$. We have seen an enhancement of this effect in cells pretreated with $2 \mathrm{mM}$ of sodium tetraborate $\left(80 \mathrm{ppm}{ }^{11} \mathrm{~B}\right.$, Fig. 2 panel A). This effect was registered in both A172 and Gl-Tr glioma cells (Fig. 2 panel B) in the entire range of the absorbed doses used and appeared to be most pronounced for A172 cells in the intermediate dose ( 2 -4 Gy). Thus, we clearly observe a radiosensitizing effect of sodium tetraborate on glioma cells when exposed to proton radiation. This result was confirmed by AlamarBlue cell viability assay (Fig. 2 panel C).
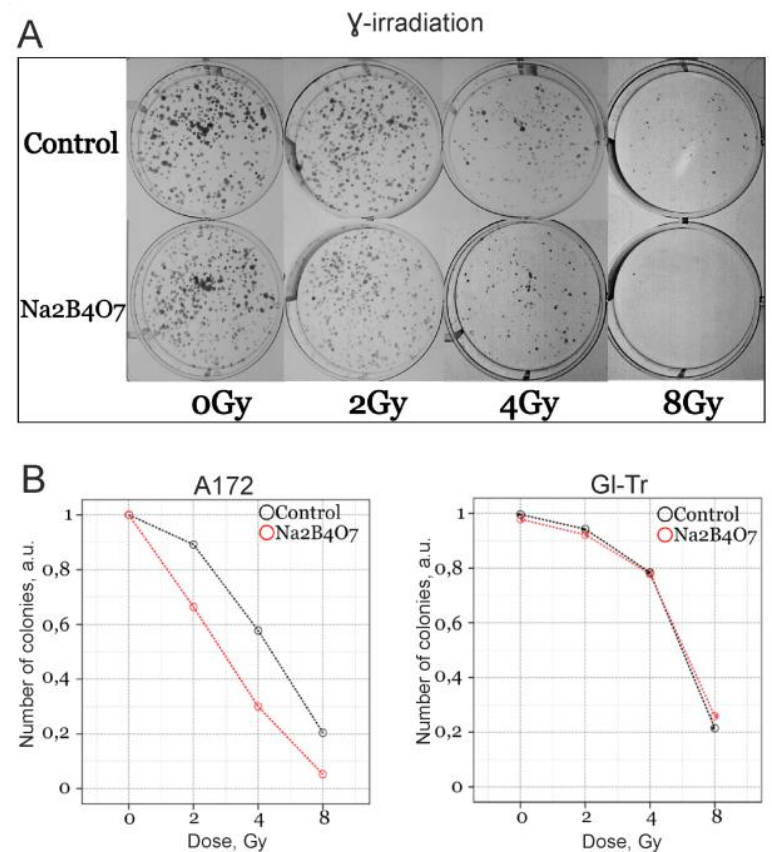

C
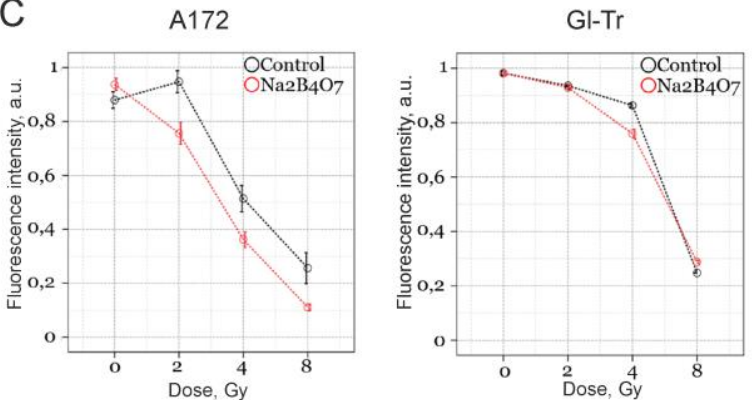

Figure 3. Effect of sodium tetraborate on cell survivability after exposure to gamma radiation at different absorbed dose.

(A) A typical colony counting test pattern for A172 cell line.

$(\mathrm{B}, \mathrm{C})$ Summary plots for clonogenic and AlamarBlue cell viability assays. Data were normalized to maximum values of the relevant control group.

We have further tested the boron-containing compound for sensitization of cells toward gamma radiation. In these experiments we have seen the enhancement of the effect of gamma radiation by preincubation with sodium tetraborate for A172 cells (Fig.3 panel A). The magnitude of this effect was close to one observed for Bragg peak protons and was also confirmed by AlamarBlue cell viability assay (Fig. 3 panels B and C). At the same time, for Gl-Tr cells no noticeable change in survivability of gamma-irradiated cells was registered upon sodium tetraborate pretreatment (Fig. 3 panels $\mathrm{B}$ and C). Thus, $\mathrm{Na}_{2} \mathrm{~B}_{4} \mathrm{O}_{7}$ 
can be a radiosensitizer to gamma irradiation of glioma cells in vitro, but its effectiveness seems to be cell line dependent.

\section{Discussion}

Our results clearly show that sodium tetraborate, being non-toxic to the normal morphology DF-2 cells at the concentrations used, can enhance the effect of proton radiation on the glioma cell lines in vitro. The fact that we observe a similar radiosensitizing effect of sodium tetraborate on A172 cells when they were subjected to gamma-radiation indicates that the decrease in the survival rate of cells irradiated after pre-incubation with the boron containing compound is likely due to their pharmacological and chemical properties rather than an increase in the absorbed dose due to ${ }^{11} \mathrm{~B}+\mathrm{p} \rightarrow 3 \alpha$ nuclear reaction. The proton capture reaction therefore may seem unlikely to play a major role in the boron enhancement of proton radiation efficacy reported earlier [10]. Notably, the earlier study [10] also reported that while the addition of ${ }^{11} \mathrm{~B}$ at a concentration of $40 \mathrm{ppm}$ led to an approximately twofold decrease in the survival rate and an increase in the frequency of chromosomal aberrations compared to irradiation without $\mathrm{BSH}$, a further increase in the concentration of ${ }^{11} \mathrm{~B}$ to $80 \mathrm{ppm}$ brought about no statistically significant changes in the survival rate and the number of chromosomal aberrations. This fact, noticed in the work of Bushmanov et al., appeared to contradict the initial hypothesis of an increase in the absorbed dose due to proton capture reaction, but rather indicated a certain threshold effect of $\mathrm{BSH}$ on the radiosensitivity of the studied tumor cells and thus supported a chemical sensibilization mechanism for the radiation effect enhancement [14].

The proton irradiation efficacy enhancement by sodium tetraborate was seen in both Gl-Tr and A172 cells, but the sensitivity of Gl-Tr to gamma radiation appeared to be unaffected by this compound. In our experiments Gl-Tr cells generally exhibited a higher resistance to ionizing radiation, which may be related to the higher fraction of cells in the "resting" Go/G1 phase of the cell cycle which are the least sensitive to radiation damage. Moreover, tetraborate appeared to further increase the fraction of Go cells in this cell line, negating the possible sensitizing effect. At the same time, Bragg peak protons appeared to be more efficient to suppress colony formation by these cells than gamma rays, possibly due to a higher LET and therefore a broader range of energies deposited within a single cell that would result in a higher frequency of events lethal to the cells resting in Go phase.

\section{CONCLUSION}

Sodium tetraborate at concentrations that are not toxic to the normal morphology epithelial cells causes significant decrease in the cell survival in two of glioma cell lines under conditions similar to those of proton radiation therapy. Similar efficiency enhancement effect was observed for gamma radiation for A172 glioma cell line, but not for Gl-Tr cell line. The mechanism of the observed radiosensitizing effect of the boron compound is unlikely to be explained by the nuclear reaction of proton capture by ${ }^{11} \mathrm{~B}$ atoms, which significance remain controversial and require further study.

Acknowledgements: The present study is a part of the research project (Order №1059) of NRC "Kurchatov Institute".

\section{REFERENCES}

1. R. Mohan, D. Grosshans, "Proton therapy - present and future," Adv. Drug Delivery Rev., vol. 109, pp. 26-44, Jan. 2017. https://doi.org/10.1016/j.addr.2016.11.006

2. X. Tian, K. Liu, Y. Hou, J Cheng, J. Zhang, "The evolution of proton beam therapy: Current and future status," Mol. Clin. Oncol., vol. 8, no. 1, pp. 15-21, Jan. 2018. https://doi.org/10.3892/mco.2017.1499

3. A. C. Begg, F. A. Stewart, C. Vens, "Strategies to improve radiotherapy with targeted drugs," Nat. Rev. Cancer, vol. 11, no. 4, pp. 239-253, Apr. 2011.

https://doi.org/10.1038/nrc3007 PMid: 21430696

4. F. Tranquart, "Radiosensitizers and radiochemotherapy in the treatment of cancer," Ultrasound in Medicine \& Biology, vol. 42, no. 2, Nov. 2015.

https://doi.org/10.1016/j.ultrasmedbio.2015.10.00 4

5. A. A. Lipengol'ts, A. A. Cherepanov, V. N. Kulakov, E. Yu. Grigor'eva, I. B. Merkulova, I. N. Sheino, "Comparison of the antitumor efficacy of bismuth and gadolinium as dose-enhancing agents in formulations for photon capture therapy," Pharm. Chem., vol. 51, pp. 783-786, Dec. 2017. https://doi.org/10.1007/s11094-017-1693-1

6. I. N. Sheino, P. V. Izhevskij, A. A. Lipengolts, V. N. Kulakov, A. A. Wagner, E. S. Sukhikh et al., "Development of binary technologies of radiotherapy of malignant neoplasms: condition and problems," Bulletin of Siberian Medicine, vol. 16, no. 3, pp. 192-209, 2017.

https://doi.org/10.20538/1682-0363-2017-3-192209

7. S. Miyatake, M. Wanibuchi, N. Hu, K. Ono, "Boron neutron capture therapy for malignant brain tumors," J. Neurooncol., vol. 149, no. 1, pp. 1-11, Aug. 2020.

https://doi.org/10.1007/s11060-020-03586-6

8. D. Yoon, J. Jung, T. Suh, "Application of proton boron fusion reaction to radiation therapy: A Monte Carlo simulation study," Appl. Phys. Lett., vol. 105, p. 223507, Dec. 2014. https://doi.org/10.1063/1.4903345

9. J. Y. Jung, D. K. Yoon, B. Barraclough, H. C. Lee, T. S. Suh, B. Lu, "Comparison between proton boron fusion therapy (PBFT) and boron neutron capture therapy (BNCT): Monte Carlo study," Oncotarget., vol. 8, no. 24, pp. 39774-39781, Feb. 2017. https://doi.org/10.18632/oncotarget.15700

10. G. A. P. Cirrone, L. Manti, D. Margarone, G. Petringa, L. Giuffrida, A. Minopoli et al., "First experimental proof of proton boron capture therapy (PBCT) to enhance proton therapy effectiveness," Sci. Rep., vol. 8, no. 1, p. 1141, Jan. 2018. https://doi.org/10.1038/s41598-018-19258-5 
11. A. Mazzone, P. Finocchiaro, S. Lo Meo, N. Colonna, "On the (un) effectiveness of proton boron capture in proton therapy," Eur. Phys. J. Plus, vol. 134, p. 361, Jul. 2019.

https://doi.org/10.1140/epjp/i2019-12725-8

12. A. Volnitskiy, T. Shtam, V. Burdakov, R. Kovalev, A. Konev, M. Filatov, "Abnormal activity of transcription factors gli in high-grade gliomas," PLoS One, vol. 14, no. 2, pp. e0211980, Feb. 2019. https://doi.org/10.1371/journal.pone.0211980 PMid: 30730955 PMCid: PMC6366868

13. S.A. Artamonov, E.M. Ivanov, N.A. Ivanov, J.S. Lebedeva, G. A. Riabov. "Numerical simulation and optimization of the variable energy 60-1000 $\mathrm{MeV}$ proton beams at PNPI synchrocyclotron for testing the radiation resistance of electronics," Phys. Part. Nuclei Lett., vol. 14, pp. 188-200, Jan. 2017.

https://doi.org/10.1134/S1547477117010046

14. A. Yu. Bushmanov, I. N. Sheino, A. A. Lipengolts, A. N. Solovev, S. N. Koryakin, "Prospects of proton therapy combined technologies in the treatment of cancer," Medical Radiology and Radiation Safety, vol. 64, no. 3, pp. 8-11, 2019.

https://doi.org/10.1371/journal.pone.0211980 